\title{
Multi-objective Optimization of Joint Power Allocation and Splitting Control for SWIPT-enabled NOMA Systems
}

DOI:

10.1109/iccnc.2019.8685620

\section{Document Version}

Accepted author manuscript

Link to publication record in Manchester Research Explorer

\section{Citation for published version (APA):}

Tang, J., Yu, Y., So, D. K. C., Chen, G., Zhang, X., \& Huang, M. (2019). Multi-objective Optimization of Joint Power Allocation and Splitting Control for SWIPT-enabled NOMA Systems. In International Conference on Computing, Networking and Communications https://doi.org/10.1109/iccnc.2019.8685620

\section{Published in:}

International Conference on Computing, Networking and Communications

\section{Citing this paper}

Please note that where the full-text provided on Manchester Research Explorer is the Author Accepted Manuscript or Proof version this may differ from the final Published version. If citing, it is advised that you check and use the publisher's definitive version.

\section{General rights}

Copyright and moral rights for the publications made accessible in the Research Explorer are retained by the authors and/or other copyright owners and it is a condition of accessing publications that users recognise and abide by the legal requirements associated with these rights.

\section{Takedown policy}

If you believe that this document breaches copyright please refer to the University of Manchester's Takedown Procedures [http://man.ac.uk/04Y6Bo] or contact uml.scholarlycommunications@manchester.ac.uk providing relevant details, so we can investigate your claim.

\section{OPEN ACCESS}




\title{
Multi-objective Optimization of Joint Power Allocation and Splitting Control for SWIPT-enabled NOMA Systems
}

\author{
Jie Tang*†, Yu Yu*, Daniel So ${ }^{\ddagger}$, Gaojie Chen ${ }^{\S}$, Xiuyin Zhang*, Mo Huang* \\ ${ }^{*}$ School of Electronic and Information Engineering, South China University of Technology, China \\ ${ }^{\dagger}$ The State Key Laboratory of Integrated Services Networks, Xidian University, UK \\ ${ }^{\ddagger}$ School of Electrical and Electronic Engineering, University of Manchester, UK \\ ${ }^{\S}$ Digital Communications and Intelligent Sensing Research Group, University of Leicester, China
}

\begin{abstract}
In this paper, we investigate joint power allocation and splitting control in a SWIPT-enabled NOMA Systems applied with power splitting technique, with an aim to optimize the transmission rate and the total harvested energy simultaneously whilst satisfying the minimum transmission rate and minimum harvested energy of each user. These two conflicting objectives make the formulated optimization problem a constrained multiobjective optimization (MOO) problem, which is difficult to solve. To deal with this, we define a new objective function by summing the weighted values of the transmission rate achieved by information decoding (ID) and the transformed throughput from energy harvesting (EH) and transform the original MOO problem into a single-objective optimization problem. To tackle the formulated nonconvex problem, we decouple the optimization problem into two convex subproblems and solve them iteratively. Numerical results demonstrate that significant performance gain can be achieved by adopting the proposed algorithm.
\end{abstract}

Index Terms-Non-orthogonal multiple access (NOMA), power splitting, resource allocation, simultaneous wireless information and power transfer (SWIPT).

\section{INTRODUCTION}

The rapid rise in traffic demands has driven the incentive for the research and development of 5G [1], which will need to deliver high spectral efficiency (SE) to pave the way for future ultra high-rate applications and the Internet-of-Things (IoT) era. Since the conventional orthogonal multiple access (OMA) techniques are not able to meet the demand of higher spectral efficiency, non-orthogonal multiple access (NOMA) has emerged as a candidate for $5 \mathrm{G}$ networks for its superior SE performance [2], [3]. In particular, Yuya Saito and Benjebbour in [2] showed that overall cell throughput, cell-edge user throughput, and the degree of proportional fairness of NOMA are all superior to that for OMA. In [3], Chen et al. showed that NOMA offered a better sum rate performance when considering user fairness.

Meanwhile, 5G application scenarios such as automation factory and smart city require long life battery to support the demand of high data rate services. Simultaneous wireless information and power transfer (SWIPT), a technique that achieves the parallel transmission of information and energy has emerged recently to prolong battery-comstrained communication devices' operation time. An information-theoretic study on SWIPT was first investigated in [4]. Since the nodes cannot harvest energy and receive/transmit information simultaneously in practice, two new receiver structures namely time switching (TS) scheme and power splitting (PS) scheme were developed [5]. Zhou et al. in [6] studied the weighted sum-rate maximization problem in a multiuser orthogonal frequency division multiplexing (OFDM) system that considered both TS and PS scheme. The capacity maximization problem and the harvested energy optimization problem have also been studied in [7] and [8], respectively.

Due to the immense potential SWIPT and NOMA, the combination of these two techniques has aroused great interest. In [9], the proportional fairness was maximized for SWIPT NOMA systems, where the harvest-then-transmit scheme was adopted in the receiver side. Furthermore, the work in [10] considered both proportional fairness improvement and data rate per-user optimization for SWIPT NOMA systems. It was demonstrated that the system performance could be significantly improved through the integration of SWIPT and NOMA. In [11], a novel cooperative SWIPT NOMA protocol was proposed in which near NOMA users that were close to the source act as energy harvesting relays to help far NOMA users. These previous literature on SWIPT-enabled NOMA systems aim to maximize either harvested energy [8] or transmission rate [9]-[11]. Because these two optimization objectives are contradictory to each other, not only is the trade-off between these two objectives a problem worth investigating, jointly optimizing them can best exploit the available resource for SWIPT NOMA systems.

The main difference of our work with the previous works mentioned above is that we aim to maximize the transmission rate and the total harvested energy simultaneously. In this paper, we investigate joint power allocation and splitting control under the constraints on the minimum transmission rate and harvested energy of each user. To solve the proposed multi-objective optimization (MOO) problem, we transform the harvested power to throughput and reformulate the original problem in to a single-objective optimization (SOO) problem with weighted sum method. Then decouple the nonconvex SOO problem into two convex subproblems that are easily solved. 


\section{System Model}

We consider a downlink NOMA system with one base station (BS) and $N$ users. We assume that the instantaneous channel state information (CSI) of each user is perfectly known at the BS and the channel power gain is denoted by $\left|h_{n}\right|^{2}$. Without loss of generality, the channel gains can be sorted as $\left|h_{1}\right|^{2} \leq\left|h_{2}\right|^{2} \leq \cdots \leq\left|h_{N}\right|^{2}$.According to the principle of NOMA [2], the transmit signal $x$ can be expressed as

$$
x=\sum_{n=1}^{N} \sqrt{p}_{n} x_{n},
$$

where $x_{n}, p_{n}$ are respectively the message and assigned power of user $n$. The transmitting power is limited by

$$
\sum_{n=1}^{N} p_{n} \leq P
$$

We assume that all the users are capable of harvesting energy from RF signals and perform SWIPT by applying PS technique. Let $\rho_{n}\left(0 \leq \rho_{n} \leq 1\right)$ denotes the fraction of transmission power allocated to user $n$ for ID. With successive interference cancellation (SIC) operation, each user $U_{i}$ will detect and remove the message of $U_{j}$ from its observed signal, for all $i>j$ [12]. With the power split to the information receiver, the achievable transmission rate for user $n$ can be expressed as

$$
R_{n}^{I D}=\log _{2}\left(1+\frac{\rho_{n}\left|h_{n}\right|^{2} p_{n}}{\rho_{n}\left|h_{n}\right|^{2} \sum_{i=n+1}^{N} p_{i}+\sigma^{2}}\right) .
$$

And the harvested energy at the receiver of user $n$ is given by

$$
e_{n}=\eta\left(1-\rho_{n}\right)\left|h_{n}\right|^{2} \sum_{n=1}^{N} p_{n}
$$

where $\eta$ represents the energy conversion efficiency. Thus, the total transmission rate and total harvested energy of the system can be written as $R_{I D}=\sum_{n=1}^{N} R_{n}^{I D}$ and $E=\sum_{n=1}^{N} e_{n}$.

\section{Problem Formulation}

In this section, we formulate the equivalent-sum-rate (ESR) maximization problem to maximize the total transmission rate and the total harvested energy simultaneously in terms of power allocation and splitting control. Besides, we consider the minimum transmission rate and the minimum harvested energy constraints of each user.

These two conflicting objectives, $R_{I D}$ and $E$, make our optimization problem a constrained MOO problem, which is often solved by combining its multiple objectives into a singleobjective scalar function. This approach is in general known as the weighted sum or scalarization method [13]. It can be proved that the maximizer of this SOO is an efficient solution for the original MOO [14], i.e., its image belongs to the Pareto curve. However, the unit for $R_{I D}$ is bit/s/Hz while the unit for $E$ is Watts, and hence it is inappropriate to directly sum them up together. In practice, the harvested power is usually stored in the battery and used to support the transmission in the following time slots, i.e., uplink transmission. On the basis of this, we transform the harvested energy into throughput using Shannon formula and define a novel paradigm by summing the weighted values of the transmission rate achieved by ID and the transformed throughput from $\mathrm{EH}$.

First, we define $R_{n}^{E H}$ to represent the achievable rate that transformed from the harvested energy of user $n$ as

$$
R_{n}^{E H}=\log _{2}\left(1+\frac{\zeta e_{n}}{\sigma^{2}}\right),
$$

where $\zeta$ denotes the efficiency converting from battery power to $\mathrm{RF}$, and the total data rates is $R_{E H}=\sum_{n=1}^{N} R_{n}^{E H}$. Then we formulate the objective function of our optimization problem as

$$
R=R_{I D}+\beta R_{E H},
$$

where $\beta$ is the weight of $R_{E H}$ and $R$ is defined as the equivalent-sum-rate of the system. It should be noted that both $R_{I D}$ and $R_{E H}$ are non-decreasing with respect to the transmit power, and given that we aim to maximize the ESR, the BS is set to use the maximum power. Considering the minimum transmission rate targets and the minimum transferred energy demands, the ESR maximization problem can be mathematically formulated as follows

$$
\begin{aligned}
& \text { P1: } \max _{\mathbf{p}, \boldsymbol{\rho}} R(\mathbf{p}, \boldsymbol{\rho}) \\
& \text { s.t. } R_{n}^{I D} \geq R_{\text {min }}, \quad \forall n=1,2, \cdots, N \text {, } \\
& e_{n} \geq E_{\text {min }}, \quad \forall n=1,2, \cdots, N \text {, } \\
& \sum_{n=1}^{N} p_{n}=P \text {, } \\
& \mathbf{p}>\mathbf{0} \text {, } \\
& \mathbf{0}<\boldsymbol{\rho}<\mathbf{1},
\end{aligned}
$$

where $\mathbf{p}=\left(p_{1}, p_{2}, \cdots, p_{N}\right)^{T}, \boldsymbol{\rho}=\left(\rho_{1}, \rho_{2}, \cdots, \rho_{N}\right)^{T}, R_{\text {min }}$ and $E_{\text {min }}$ are the minimum transmission rate requirements and the minimum harvested energy requirements, respectively.

The considered ESR maximization problem, with joint power allocation and splitting control in the presence of interuser interference, is non-convex. The solution is therefore non-trivial and cannot be obtained directly. It is obvious that this exhaustive search method incurs intensive computational complexity in the number of users. As a result, an efficient resource allocation strategy are developed by decomposing the optimization problem into two subproblems and solve iteratively.

\section{Iterative Resource Allocation Scheme}

In this section, we first decompose the optimization problem into two subproblems, one of which is optimizing $\mathbf{p}$ with fixed $\rho$ and the other is optimizing $\rho$ with fixed $\mathbf{p}$, and then we propose a suboptimal resource allocation algorithm by solving them iteratively .

\section{A. Optimal PS Ratio}

To find the optimal PS ratio with fixed power allocation, the corresponding subproblem can be mathematically formulated 
as follows

$$
\begin{array}{cl}
\text { P2: } & \max _{\boldsymbol{\rho}} R(\boldsymbol{\rho}) \\
\text { s.t. } & (7 b)(7 c)(7 f) .
\end{array}
$$

The Hessian matrix of $R(\boldsymbol{\rho})$ can be given as

$$
\frac{d^{2} R(\boldsymbol{\rho})}{d \boldsymbol{\rho}^{2}}=\left[\begin{array}{cccc}
\frac{d^{2} R(\boldsymbol{\rho})}{d \rho_{1}^{2}} & 0 & \cdots & 0 \\
0 & \frac{d^{2} R(\boldsymbol{\rho})}{d \rho_{2}^{2}} & \cdots & 0 \\
\vdots & \vdots & \ddots & \vdots \\
0 & 0 & \cdots & \frac{d^{2} R(\boldsymbol{\rho})}{d \rho_{N}^{2}}
\end{array}\right]
$$

It is easy to calculate that $\frac{d^{2} R(\boldsymbol{\rho})}{d \rho_{n}^{2}}<0, \forall n=1,2, \cdots, N$, which reveals $\frac{d^{2} R(\rho)}{d \rho^{2}}$ is a negative semi-definite matrix and thus $R(\boldsymbol{\rho})$ is a concave function with respect to $\boldsymbol{\rho}$. Here we rewrite the objective function as

$$
\begin{aligned}
R & =\sum_{n=1}^{N} R_{n}^{I D}+\beta \sum_{n=1}^{N} R_{n}^{E H} \\
& =\sum_{n=1}^{N}\left(R_{n}^{I D}+\beta R_{n}^{E H}\right)=\sum_{n=1}^{N} R_{n},
\end{aligned}
$$

where $R_{n}=R_{n_{2}}^{I D}+\beta R_{n}^{E H}$ denotes the equivalent-data-rate of user $n$. Since $\frac{d^{2} R(\boldsymbol{\rho})}{d \rho_{i} d \rho_{i}}=0, \forall i \neq j$, the PS ratios of users are independent of each other, the maximization of ESR for all the users is equivalent to the maximization of the individual equivalent-data-rate of each user. Consequently, problem P2 can be divided into $N$ parallel subproblems which can be solved using the same solution. Based on this observation, we propose an efficient algorithm to solve this problem. We individually maximize $R_{n}\left(\rho_{n}\right)$ subject to the constraints of each user, and then unify a solution set from all subproblems. Generally, the subproblems of $\mathbf{P} \mathbf{2}$ are given as follows

$$
\begin{gathered}
\text { P3: } \max _{\rho_{n}, n=1,2, \cdots, N} R_{n}\left(\rho_{n}\right) \\
\text { s.t. } \quad(7 b)(7 c)(7 f) .
\end{gathered}
$$

Since $\frac{d^{2} R(\rho)}{d \rho_{n}^{2}}<0, R_{n}$ is strictly concave with respect to $\rho_{n}$. It means that there is an unique root of the equation $\frac{d R\left(\rho_{n}\right)}{d \rho_{n}}=0$ to maximize $R_{n}$, which is detailed expressed as

$$
\begin{aligned}
\frac{d R\left(\rho_{n}\right)}{d \rho_{n}}= & \frac{\left|h_{n}\right|^{2} p_{n} \sigma^{2}}{\ln 2\left(\rho_{n}\left|h_{n}\right|^{2} \sum_{i=n}^{N} p_{i}+\sigma^{2}\right)\left(\rho_{n}\left|h_{n}\right|^{2} \sum_{i=n+1}^{N} p_{i}+\sigma^{2}\right)} \\
& +\frac{-\beta \eta\left|h_{n}\right|^{2} P}{\ln 2\left(\eta\left(1-\rho_{n}\right)\left|h_{n}\right|^{2} P+\sigma^{2}\right)}=0 .
\end{aligned}
$$

We denote the root as $\hat{\rho}$. According to the constraints (11b), the value of $\rho_{n}$ should be limited as

$$
\rho_{n}^{\min } \leq \rho_{n} \leq \rho_{n}^{\max },
$$

where $\rho_{n}^{\text {min }}=\frac{\left(2^{R_{m i n}}-1\right) \sigma^{2}}{\left|h_{n}\right|^{2} p_{n}-\left(2^{R_{m i n}}-1\right)\left|h_{n}\right|^{2} \sum_{i=n+1}^{N} p_{i}}>0$ ensures that the power split to ID can meet the user's minimum rate requirement, and $\rho_{n}^{\max }=1-\frac{E_{\min }}{\eta\left|h_{n}\right|^{2} P}<1$ ensures the power fed to EH is able to satisfy the minimum harvested energy requirement. To meet the QoS requirements, the optimal PS ratio of user $n$ is finally given by

$$
\rho_{n}^{*}=\left\{\begin{array}{l}
\rho_{n}^{\min }, \quad \hat{\rho}_{n}<\rho_{n}^{\min } \\
\hat{\rho}_{n}, \quad \rho_{n}^{\min } \leq \hat{\rho}_{n} \leq \rho_{n}^{\max } \\
\rho_{n}^{\max }, \quad \hat{\rho_{n}}>\rho_{n}^{\max }
\end{array} .\right.
$$

Thus the optimal solution of is $\mathbf{P} 2$ is $\boldsymbol{\rho}^{*}=\left(\rho_{1}^{*}, \rho_{2}^{*}, \cdots, \rho_{N}^{*}\right)^{T}$.

\section{B. Optimal Power Allocation}

With fixed PS ratios, the corresponding subproblem to find an efficient power allocation algorithm can be mathematically formulated as

$$
\begin{array}{cc}
\text { P4: } & \max _{\mathbf{p}} R(\mathbf{p}) \\
\text { s.t. } & (7 b)(7 d)(7 e) .
\end{array}
$$

To solve the above problem, a relationship between $R(\mathbf{p})$ and $\mathbf{p}$ is derived as in the following proposition.

Proposition I. With fixed transmit power and PS ratios, the objective function of problem $\boldsymbol{P 4}$ is a concave function with respect to $\boldsymbol{p}$ if and only if $\rho_{1}\left|h_{1}\right|^{2} \leq \rho_{2}\left|h_{2}\right|^{2} \leq \cdots \leq \rho_{N}\left|h_{N}\right|^{2}$.

Proof: The proof can be obtained by proving the Hessian matrix of $R(\mathbf{p})$ is negative semi-definite, which is omitted due to space limitation.

Here we assume $\rho_{1}\left|h_{1}\right|^{2}<\rho_{2}\left|h_{2}\right|^{2}<\cdots<\rho_{N}\left|h_{N}\right|^{2}$, and then the objective function of $\mathbf{P 4}$ is concave according to Proposition I. As the result, problem $\mathbf{P 4}$ is convex since (7b) is convex and (7d), (7e) are linear, which means it can be solved by standard numerical methods such as interior point method. Although the convex programming approach is numerically stable, its computational complexity depends on the number of optimizing variables, which can be problematic if the number of users is large. To reduce the complexity, we use Lagrange dual decomposition method which is more efficient. The Lagrangian function is given by

$$
\mathcal{L}(\mathbf{p}, \boldsymbol{\lambda}, \mu)=-R(\mathbf{p})+\sum_{n=1}^{N} \lambda_{n} f_{n}+\mu h,
$$

where $\boldsymbol{\lambda}$ is the Lagrange multiplier vector with elements $\lambda_{n} \geq$ 0 corresponds to the constraints in (7b), $\mu \geq 0$ is the Lagrange multiplier corresponds to the constraint (7d). And

$$
\begin{gathered}
f_{n}=R_{\min }-\log _{2}\left(1+\frac{\rho_{n}\left|h_{n}\right|^{2} p_{n}}{\rho_{n}\left|h_{n}\right|^{2} \sum_{i=n+1}^{N} p_{i}+\sigma^{2}}\right), \\
h=\sum_{n=1}^{N} p_{n}-P .
\end{gathered}
$$

The Karush-Kuhn-Tucker (KKT) conditions of problem P4 is given by

$$
\begin{aligned}
& \frac{d \mathcal{L}(\mathbf{p}, \boldsymbol{\lambda}, \mu)}{d p_{n}}=0, \quad \forall n=1,2, \cdots, N \\
& \lambda_{n} f_{n}=0, \quad f_{n} \leq 0, \quad \lambda_{n} \geq 0, \quad \forall n=1,2, \cdots, N \\
& \mu h=0, \quad h=0, \quad \mu \geq 0
\end{aligned}
$$


and for a given user $\bar{n}, \frac{d \mathcal{L}(\mathbf{p}, \boldsymbol{\lambda}, \mu)}{d p_{\bar{n}}}$ is detailed as (20) in the next page.

According to (19a), we obtain $\frac{d \mathcal{L}(\mathbf{p}, \boldsymbol{\lambda}, \mu)}{d p_{n}}-\frac{d \mathcal{L}(\mathbf{p}, \boldsymbol{\lambda}, \mu)}{d p_{n-1}}=0$, which is analyzed in (21) in the next page. And then we have

$$
\begin{aligned}
& \frac{\lambda_{n-1} \rho_{n-1}\left|h_{n-1}\right|^{2}}{\ln 2\left(\rho_{n-1}\left|h_{n-1}\right|^{2} \sum_{i=n-1}^{N} p_{i}+\sigma^{2}\right)}-\frac{\lambda_{n} \rho_{n}\left|h_{n}\right|^{2}}{\ln 2\left(\rho_{n}\left|h_{n}\right|^{2} \sum_{i=n}^{N} p_{i}+\sigma^{2}\right)} \\
& =\frac{\rho_{n}\left|h_{n}\right|^{2}}{\ln 2\left(\rho_{n}\left|h_{n}\right|^{2} \sum_{i=n}^{N} p_{i}+\sigma^{2}\right)}-\frac{\rho_{n-1}\left|h_{n-1}\right|^{2}}{\ln 2\left(\rho_{n-1}\left|h_{n-1}\right|^{2} \sum_{i=n}^{N} p_{i}+\sigma^{2}\right)}
\end{aligned}
$$$$
>0 \text {. }
$$

With $\rho_{1}\left|h_{1}\right|^{2}<\rho_{2}\left|h_{2}\right|^{2}<\cdots<\rho_{N}\left|h_{N}\right|^{2}$, it is easy to obtain $\lambda_{n}>0$ when $n=1,2, \cdots, N-1$. This reveals that the constraints in (7b) hold with equality for $n=1,2, \cdots, N-1$. With $\lambda_{N}>0, f_{N}$ must be 0 . In this case, all the users' achievable transmission rates are just satisfy the minimum QoS requirements. Thus, we can calculate the total power consumption, denoted as $P_{\text {min }}$, which indicates the minimum power that satisfies all users' QoS requirements. We suppose $P \geq P_{\min }$ in this work for a practical configuration. With $\lambda_{N}=0, f_{N}$ can be negative, it indicates that the achievable transmission rate of the user with best channel gain can further improve under the premise of meeting the minimum rate requirements.

For the case that $P=P_{\text {min }}$, to obtain the optimal power allocation, we only need to set $R_{n}^{I D}=R_{\min }$ for all the users, and $p_{n}$ can be sequentially determined in the order $n=N, N-1, \cdots, 1$.

For the case that $P>P_{\text {min }}$, the constraints in (7b) hold with equality for $n=1,2, \cdots, N-1$ except $n=N$. Hence, the BS transmits to user with best channel condition using the remaining power $P-\sum_{i=1}^{N-1} p_{i}$. Therefore, we can conclude the optimal power allocation solution for problem P4 as

$$
\left\{\begin{array}{l}
p_{n}=\frac{2^{R_{\min }-1}}{2^{R_{\min }}}\left(P-\sum_{i=1}^{n-1} p_{i}+\frac{\sigma^{2}}{\rho_{n}\left|h_{n}\right|^{2}}\right), n=1,2, \cdots, N-1 \\
p_{N}=P-\sum_{n=1}^{N-1} p_{n}
\end{array}\right.
$$

The proposed power allocation scheme indicates that more resources are allocated to the user with best channel condition in order to further improve the system, and similar results are observed in the conventional NOMA systems [15].

\section{Joint Optimization of Power Allocation and PS Ratio}

The joint optimization of power allocation and power splitting ratios leads our problem non-convex and it is very challenging to solve. But on the basis that the objective function is concave on $\mathbf{p}$ and $\rho$ respectively, we are able to find the suboptimal solution by solving the two subproblems iteratively, as described below.

Begin with finding the optimal PS ratios, we first initial the PS ratios with same value and determine the power allocation according to (23). With fixed p, we can get the optimal PS ratios with (12) and (14). Before going on the next step to find
TABLE I: The proposed suboptimal resource allocation algorithm

I. Initial the PS ratio $\rho^{(1)}, 0<\rho_{1}^{(1)}=\cdots=\rho_{N}^{(1)}<1$, set $R_{\boldsymbol{\rho}}^{(1)}=N R_{\min }+\beta N \log _{2}\left(1+\frac{\zeta E_{\min }}{\sigma^{2}}\right), \delta=10^{-5}$ as the maximum tolerance;

II. Solve the problem P4 using (23) with $\boldsymbol{\rho}^{(1)}$, record the power allocation as $\mathbf{p}^{(1)}$, ESR as $R_{\mathbf{p}}^{(1)}$;

\section{III.REPEAT}

Solve the problem $\mathbf{P 3}$ using (12)(14) with $\mathbf{p}^{(t-1)}$, record the PS ratio as $\boldsymbol{\rho}^{(t)}, \operatorname{ESR} R_{\boldsymbol{\rho}}^{(t)}$, reorder $\rho_{n}^{*}\left|h_{n}\right|^{2}$ in the ascending order;

IF $R_{\boldsymbol{\rho}}^{(t)}-R_{\mathbf{p}}^{(t-1)}>\delta$ :

Solve the problem P4 using (23) with $\boldsymbol{\rho}^{(t)}$, record the power allocation as $\mathbf{p}^{(t)}$, ESR as $R_{\mathbf{p}}^{(t)}$;

$$
\begin{aligned}
& \text { IF } R_{\mathbf{p}}^{(t)}-R_{\boldsymbol{\rho}}^{(t)}>\delta \text { : } \\
& \quad \text { Convergence }=\text { FALSE; } \\
& \text { ELSE }
\end{aligned}
$$

$$
\text { Convergence }=\text { TRUE; }
$$

$$
\text { RED }
$$

ELSE

$$
\text { Convergence }=\text { TRUE; }
$$$$
\text { RETURN } \mathbf{p}^{*}=\mathbf{p}^{(t-1)}, \boldsymbol{\rho}^{(*)}=\boldsymbol{\rho}^{(t-1)}, R^{*}=R_{\mathbf{p}}^{(t-1)}
$$$$
\text { END }
$$

UNTIL Convergence $=$ TRUE.

the optimal power allocation scheme, we multiply the optimal PS ratios with the corresponding channel gains and reordering them in the ascending order, so that the optimization scheme we propose for subproblem P4 can be feasible. The two subproblems will be solved iteratively until the ERS converges. The complete algorithm is summarized in Table I.

\section{SIMULATION RESULTS AND DISCUSSION}

. In this section, simulation results are provided in order to validate the performance of the proposed MOO model and algorithm in the SWIPT-enabled NOMA system. It is assumed that there are 6 users uniformly-distributed in the coverage area of BS. All the results are obtained from various random locations of the users with identical and independent Rayleigh block fading channels and Log-Normal shadowing with standard deviation of $8 \mathrm{~dB}$. For the path loss, we here apply the generic channel model where the path-loss is given by $\left(\frac{d_{0}}{d}\right)^{v}$. Particularly, $d$ and $d_{0}$ are respectively representing the distance between the transmitter and the receiver and the reference distance, and the path-loss exponent $v$ is set to 2.5. In addition, the $\mathrm{EH}$ efficiency $\eta$ and conversion efficiency $\zeta$ are set to be $10 \%$, and the preference coefficient (combining weights) $\beta$ is set to 0.1 . In order to guarantee QoS requirements for each user, the minimum transmission rate constraint per user is set to $2 \mathrm{bit} / \mathrm{s} / \mathrm{Hz}$, and the minimum harvested energy per user is set to $0.1 \mathrm{~W}$. Note that, in all our simulations, if any user whose rate or harvested energy does not meet the requirements, the ESR is set to zero. 


$$
\begin{aligned}
\frac{d \mathcal{L}(\mathbf{p}, \boldsymbol{\lambda}, \mu)}{d p_{\bar{n}}}= & \sum_{n=1}^{\bar{n}-1} \lambda_{n} \frac{\rho_{n}^{2}\left|h_{n}\right|^{4} p_{n}}{\ln 2\left(\rho_{n}\left|h_{n}\right|^{2} \sum_{i=n}^{N} p_{i}+\sigma^{2}\right)\left(\rho_{n}\left|h_{n}\right|^{2} \sum_{i=n+1}^{N} p_{i}+\sigma^{2}\right)}-\lambda_{\bar{n}} \frac{\rho_{\bar{n}}\left|h_{\bar{n}}\right|^{2}}{\ln 2\left(\rho_{\bar{n}}\left|h_{\bar{n}}\right|^{2} \sum_{i=\bar{n}}^{N} p_{i}+\sigma^{2}\right)} \\
& -\sum_{n=1}^{\bar{n}-1}\left(\frac{\rho_{n+1}\left|h_{n+1}\right|^{2}}{\ln 2\left(\rho_{n+1}\left|h_{n+1}\right|^{2} \sum_{i=n+1}^{N} p_{i}+\sigma^{2}\right)}-\frac{\left.\rho_{n}\right|^{2}}{\ln 2\left(\rho_{n}\left|h_{n}\right|^{2} \sum_{i=n+1}^{N} p_{i}+\sigma^{2}\right)}\right)-\frac{\rho_{1}\left|h_{1}\right|^{2}}{\ln 2\left(\rho_{1}\left|h_{1}\right|^{2} \sum_{i=1}^{N} p_{i}+\sigma^{2}\right)}+\mu .
\end{aligned}
$$

$$
\begin{aligned}
\frac{d \mathcal{L}(\mathbf{p}, \boldsymbol{\lambda}, \mu)}{d p_{n}}-\frac{d \mathcal{L}(\mathbf{p}, \boldsymbol{\lambda}, \mu)}{d p_{n-1}}= & \frac{\rho_{n-1}\left|h_{n-1}\right|^{2}}{\ln 2\left(\rho_{n-1}\left|h_{n-1}\right|^{2} \sum_{i=n}^{N} p_{i}+\sigma^{2}\right)}-\frac{\rho_{n}\left|h_{n}\right|^{2}}{\ln 2\left(\rho_{n}\left|h_{n}\right|^{2} \sum_{i=n}^{N} p_{i}+\sigma^{2}\right)} \\
& +\frac{\lambda_{n-1} \rho_{n-1}\left|h_{n-1}\right|^{2}}{\ln 2\left(\rho_{n-1}\left|h_{n-1}\right|^{2} \sum_{i=n-1}^{N} p_{i}+\sigma^{2}\right)}-\frac{\lambda_{n} \rho_{n}\left|h_{n}\right|^{2}}{\ln 2\left(\rho_{n}\left|h_{n}\right|^{2} \sum_{i=n}^{N} p_{i}+\sigma^{2}\right)}
\end{aligned}
$$

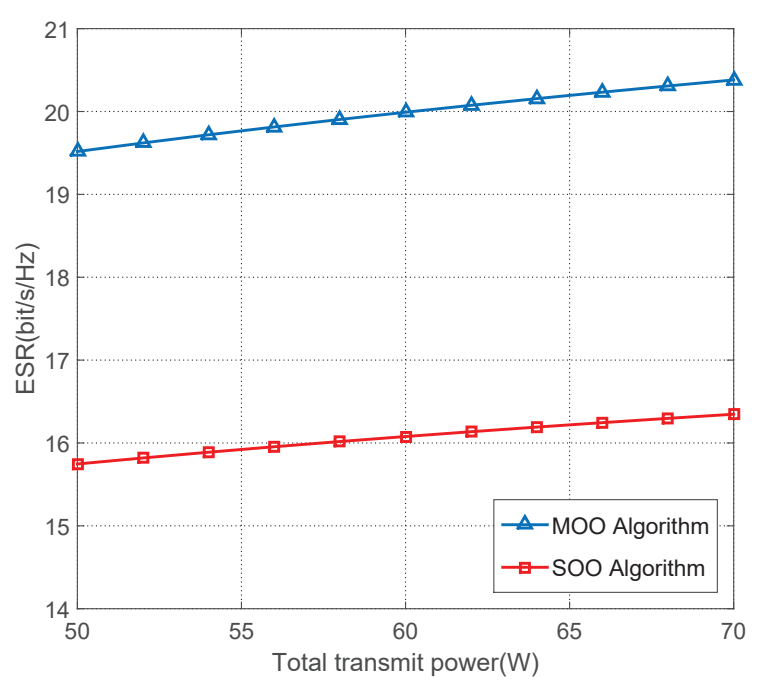

Fig. 1: ESR versus total transmit power.

Fig. 1 shows the ESR versus the total transmit power of the BS. It explicit that the performance of SWIPT-enabled NOMA system becomes better with more transmit power is provided. In order to depict the performance gain of the proposed MOO model, we compare it with the SOO problem which only maximizes the total transmission rate under the same constraints condition. It has been shown in Fig.1 that the gains in terms of ESR that our proposed MOO model can offer compared to the algorithm that only considers the optimization of transmission rate are greater. Thus, it is clear that the simultaneous maximization of total transmission rate and harvested energy can get a more effective resource allocation scheme.

We next analyse the impact of the number of users to the maximum equivalent-sum-rate. In Fig. 2, the number of users is set to 4, 5 and 6. As Fig. 2 shows, the ESR increases with the number of users when the total transmit power is large

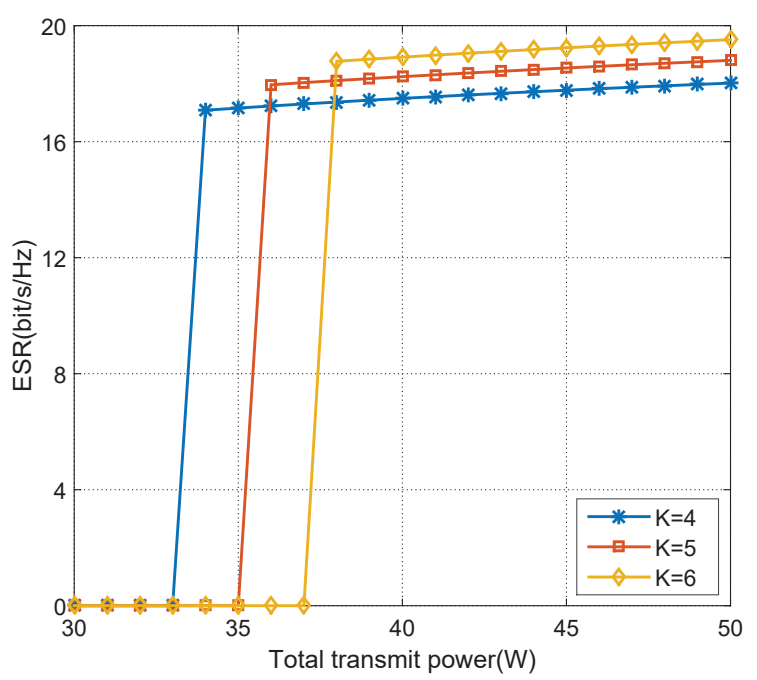

Fig. 2: ESR versus total transmit power with different number of users.

enough. This is because a higher diversity gain is offered when more users are served simultaneously. But with small P, ESR becomes zero as $\mathrm{K}$ increases. The reason is the limited power can not meet the QoS requirements of all users when the number of uses is too large. It makes no sense to improve the total performance of the system with individual's QoS requirements can not be guaranteed.

In the next simulations, we respectively analyse the impact of the minimum transmission rate and minimum harvested energy requirements to ESR with different number of users. To satisfy higher QoS requirements, here the power budget is set to $P=60 W$. As it can be seen from Fig. 3 and Fig. 4, with fixed number of users, the ESR remains nearly unchanged up to a particular minimum transmission rate and harvested energy constraints, but decreases thereafter. It is because more resource need to be allocated to the users with poor channel condition so as to meet the increasing 


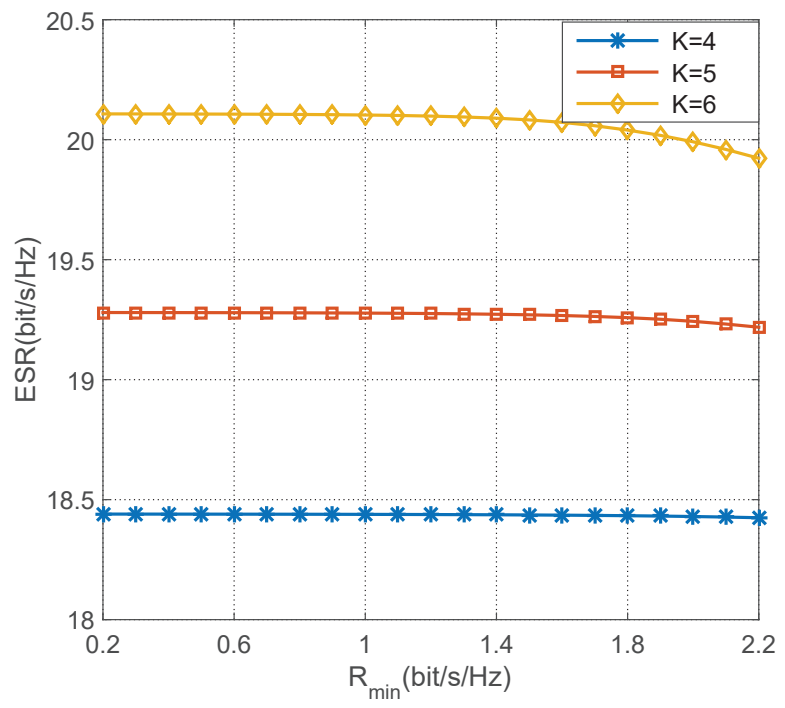

Fig. 3: ESR versus minimum transmission rate requirement with different number of users.

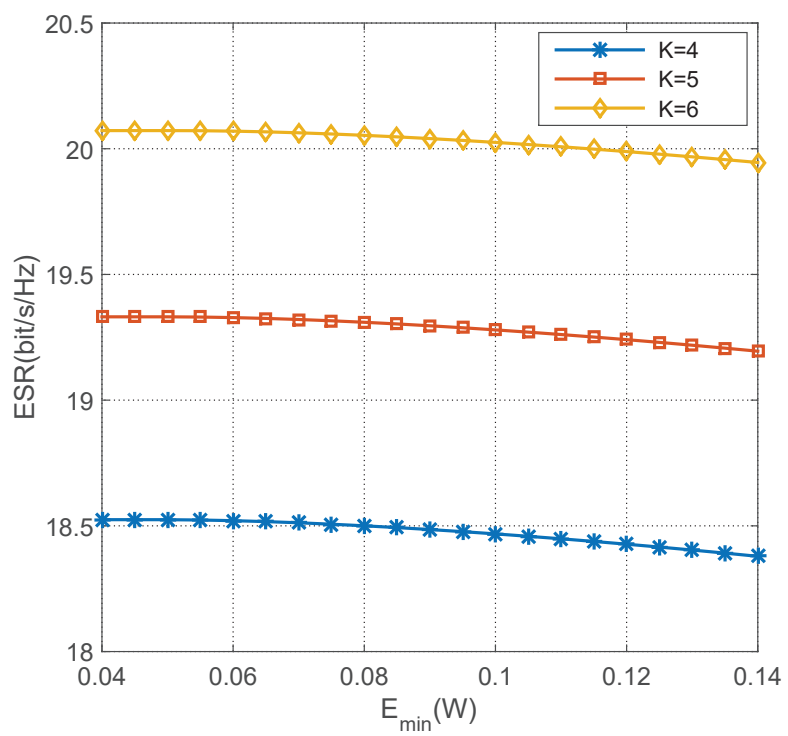

Fig. 4: ESR versus minimum harvested energy requirement with different number of users.

QoS requirements, thus degrades the overall performance of the system. Taking the number of users into consideration, the maximum equivalent-sum-rate is increasing with the number of users under the same QoS requirements, and this improvement becomes smaller with increasing users. With the limited transmit power at the BS, although more users can enhance the total ESR, meeting the QoS requirements of all the users will increase the burden of the system. It is obvious that the more users, the more quickly ESR reduces when $R_{\text {min }}$ improves.

\section{Vi. Conclusion}

This paper investigates the joint optimization of the conflicting objectives on total transmission rate and harvested energy for SWIPT-enabled NOMA systems. By considering the reverse link capacity as a function of harvested energy and introduce a weighted coefficient, we transform the constrained MOO problem to a SOO problem. This non-convex problem is solved by decoupling into two sub-problems with iterative solution. From the simulation results, the ESR increases with more transmit power, and there is a minimum power to meet all the users' QoS requirements. More importantly, compared to the optimization only respect of transmission rate, it has shown that significant performance gain can be achieved by considering both transmission rate and harvested energy simultaneously. Furthermore, the QoS requirements of individual users and the number of users have substantial impact on the system's ESR.

\section{REFERENCES}

[1] H. Zhang, Y. Dong, J. Cheng, M. J. Hossain, and V. C. M. Leung, "Fronthauling for 5G LTE-U ultra dense cloud small cell networks," IEEE Wireless Commun., vol. 23, no. 6, pp. 48 - 53, Dec. 2016.

[2] Y. Saito, A. Benjebbour, Y. Kishiyama, and T. Nakamura, "System-level performance evaluation of downlink non-orthogonal multiple access (NOMA)," in Proc. IEEE Int. Symp. Personal Indoor and Mobile Radio Commun., 2013, pp. 611-615.

[3] Z. Chen, Z. Ding, X. Dai, and R. Zhang, "An optimization perspective of the superiority of NOMA compared to conventional OMA," IEEE Trans. Sig. Process., vol. 65, no. 19, pp. 5191-5202, 2017.

[4] L. R. Varshney, "Transporting information and energy simultaneously," in IEEE Int. Symp. Inf. Theory, 2008, pp. 1612-1616.

[5] R. Zhang and C. K. Ho, "MIMO broadcasting for simultaneous wireless information and power transfer," IEEE Trans. Wireless Commun., vol. 12, no. 5, pp. 1989-2001, 2013.

[6] X. Zhou, R. Zhang, and C. K. Ho, "Wireless information and power transfer in multiuser OFDM systems," IEEE Trans. Wireless Commun., vol. 13, no. 4, pp. 2282-2294, 2014.

[7] S. Luo, J. Xu, J. L. Teng, and R. Zhang, "Capacity region of miso broadcast channel for simultaneous wireless information and power transfer," IEEE Transactions on Communications, vol. 63, no. 10, pp. 3856-3868, 2015.

[8] J. Park, B. Clerckx, C. Song, and Y. Wu, "An analysis of the optimum node density for simultaneous wireless information and power transfer in ad hoc networks," IEEE Trans. Veh. Techn., vol. PP, no. 99, pp. 1-1, 2017.

[9] P. D. Diamantoulakis and G. K. Karagiannidis, "Maximizing proportional fairness in wireless powered communications," IEEE Wireless Commun. Lett., vol. 6, no. 2, pp. 202-205, April 2017.

[10] P. D. Diamantoulakis, K. N. Pappi, Z. Ding, and G. K. Karagiannidis, "Wireless-powered communications with non-orthogonal multiple access," IEEE Trans. Wireless Commun., vol. 15, no. 12, pp. 8422-8436, Dec 2016.

[11] Y. Liu, Z. Ding, M. Elkashlan, and H. V. Poor, "Cooperative nonorthogonal multiple access with simultaneous wireless information and power transfer," IEEE Journal Sel. Areas Commun., vol. 34, no. 4, pp. 938-953, 2015.

[12] Z. Ding, Z. Yang, P. Fan, and H. V. Poor, "On the performance of non-orthogonal multiple access in $5 \mathrm{~g}$ systems with randomly deployed users," IEEE Signal Processing Letters, vol. 21, no. 12, pp. 1501-1505, 2014.

[13] J. Tang, D. K. C. So, E. Alsusa, and K. A. Hamdi, "Resource efficiency: A new paradigm on energy efficiency and spectral efficiency tradeoff," IEEE Trans. Wireless Commun., vol. 13, no. 8, pp. 46564669, Aug. 2014.

[14] G. B. L. C. A. C. Coello and D. A. V. Veldhuizen, Evolutionary Algorithms for Solving Multi-Objective Problems. New York: Springer, 2007.

[15] Z. Yang, W. Xu, C. Pan, Y. Pan, and M. Chen, "On the optimality of power allocation for noma downlinks with individual qos constraints," IEEE Commun. Lett., vol. 21, no. 7, pp. 1649-1652, July 2017. 